\title{
INFORME DE INVESTIGACIÓN
}

\section{GLADYS FORERO BOHÓRQUEZ *}

\section{LA EVALUACION DE TEXTOS PARA LA ENSEÑANZA DEL INGLES EN UN PROGRAMA DE LICENCIATURA}

\section{INTRODUCCIÓN}

Una de las tareas intrínsecas al quehacer de un profesor de inglés, es la evaluación de un texto. Evaluar un texto es confrontar sus objetivos con los del profesor, o programa interesado en usarlo, y sus contenidos y forma de presentarlos con las necesidades y preferencias de los potenciales usuarios (tanto docentes como dicentes). En este sentido, autores como Cunningsworth (1984) aseveran que evaluar un libro de texto es examinarlo a la luz de las dos principales variables del proceso educativo, a saber, los objetivos de enseñanza y el tipo de usuarios a los que va dirigida. Por su parte, Hutchinson y otro (citados por Sheldon,1987) afirman que evaluar un libro es básicamente un proceso de confrontación entre las necesidades de un contexto de ens eñanza-aprendizaje en particular y las soluciones dis ponibles.

Varias razones pueden motivar a un profesor para evaluar un libro de texto para la enseñanza del inglés como lengua extranjera: Entre otras están: que se haya oído hablar bien de él, que no se estén viendo los resultados esperados con el que se está usando en el momento o que se quiera explorar una altemativa que conduzca al mejoramiento del proceso enseñanza-aprendizaje en un deteminado curso o programa.

Si se tiene en cuenta que:

a) como afima Harmer (1983), cuando un buen número de profesores en una institución usan el mismo libro éste ejerce una poderosa influencia en el tipo de enseñanza que se dé en esa institución,

b) cuando un libro de texto para la enseñanza del inglés como lengua extranjera se usa como guía principal de un curso, éste se convierte en la herramienta principal que el alumno tiene para clarificar y/o reafimar lo que se ve en clase,

c) como lo afirma Sheldon (op. cit.), "nos guste o no, los libros de texto representan para estudiantes y profesores el corazón visible de un programa de enseñanza del inglés",

\footnotetext{
* Profesora Universidad Pedagógica Nacional 
d) se trata de un programa de nivel universitario para formar profesores de inglés como lengua extranjera,

la evaluación de un libro de texto debe fundamentarse en la revisión de al menos los siguientes criterios o variables del proceso educativo: (a) objetivos del programa de inglés, porque es indispensable que el texto de enseñanza sea compatible con los objetivos del programa interesado en usarlo; (b) tipo de usuarios al que va dirigido, en términos, por ejemplo, de la edad de los estudiantes, para saber si será más útil un texto que use fotografías para ilustrar sus temas más que dibujos o viceversa; el lugar donde viven dentro de una ciudad para saber si el texto se acomoda a sus condiciones socioeconómicas; Ios propósitos para aprender la lengua inglesa para saber si éstos están de acuerdo con los del libro; conocimientos de la lengua inglesa para saber, por ejemplo, qué número de la serie les conviene más; (c) identificación de las necesidades (a nivel de lengua inglesa) de estos usuarios, (d) procedimientos para trabajar con tareas y actividades en clase preferidos por es tos usuarios, para saber si las actividades que el texto les sugiere para que practiquen las diferentes unidades de aprendizaje son de la naturaleza de las que a ellos le ayudan a aprender, y si a juicio del evaluador, están de acuerdo con las que se necesitan para que un estudiante adquiera la habilidad para usar la lengua; (e) concepción que tiene el programa acerca de cómo se puede aprender una lengua, para saber cuál método de enseñanza de las lenguas (tradicional, estructural, o comunicativo) prima en la presentación de las unidades de aprendizaje. f) criterios de seleccion y organización de los contenidos a ser enseñados, para saber si el syllabus (organización de los contenidos) que usa el programa interesado en adoptar el libro está de acuerdo con el del texto; g) presentación de los contenidos en el libro objeto de la evaluación, para saber, por ejemplo, si son enseñables, esto es, si están divididos en pequeñas unidades de aprendizaje, de fácil asimilación por el estudiante por su claridad y coherencia y porque su contexto es motivante; y h) opiniones de los estudiantes acerca de ese texto, para saber si a ellos les gustaría estudiar el Inglés en ese determinado libro de texto.

La anterior lista de criterios está de acuerdo con la propuesta por Cunningsworth 1984 (op. cit.) que incluye aspectos tales como relacionar los materiales con los propósitos y objetivos particulares, tener en cuenta (a)para qué se necesita la lengua y asegurarse que los materiales le pemitirán a los estudiantes usarla efectivamente para ese propósito dado; (b) que aprender una lengua no es fácil y que por lo tanto el estudiante necesita que le muestren las unidades de aprendizaje en forma detallada, coherente y motivante.

La lista de criterios para la evaluación de un texto sugerida en este artículo también está de acuerdo con la afirmación de Hutchinson (1987) (op. cit.), en la que dice que es necesario que el evaluador no sólo mire los materiales sino que analice la situación enseñanza-aprendizaje para la cual se requieren estos materiales. 
En este documento se presentan los resultados de la evaluación de un libro de texto para la enseñanza del inglés en nivel intemedio, en un programa de licenciatura en Español-Inglés, de una universidad colombiana, en el siguiente orden: objetivo de la evaluación, metodología, objetivos del programa de inglés, tipo de usuarios a quienes va dirigido, identificación de las necesidades de los usuarios, concepción de "lengua" con que trabaja el programa de inglés donde se realizó la presente evaluación, el syllabus, descripción del libro ' $A$ ', descripción del libro ' $B$ ', las lecciones piloteadas de 'A' en comparación con las de 'B', procedimientos para trabajar con tareas y actividades en clase preferidos por estos usuarios, y conclusiones. Luego de un análisis cuantitativo de cada uno de los parámetros de evaluación se presentan las conclusiones y recomendaciones de la misma..

\section{1. objetivo de la evaluación}

1.1 Saber qué tan relevantes son las lecciones del libro ' $A$ ' y qué tan útiles son, en sí mismas y en comparación con el libro ' $B$ ', para estudiantes de nivel intermedio de inglés de un programa de licenciatura en Español-Inglés, a la luz de sus necesidades e intereses, y de literatura sobre evaluación de textos.

\section{2. metodología}

La evaluación se realizó con 22 estudiantes de nivel intermedio de inglés, del programa de licenciatura en Español-Inglés de una universidad colombiana. A 17 de estos estudiantes (presentes el día de la encuesta) se les aplicó un cuestionario de cinco preguntas abiertas para obtener información básica acerca del tipo de usuarios a quien va dirigido el programa de inglés.

Posteriormente, se pilotearon dos lecciones del libro para nivel intermedio de la serie ' $A$ ', , utilizando tanto los textos escritos como los de escucha de ambas lecciones. Aprovechando que estos estudiantes estaban usando como guía para la clase el libro ' $\mathrm{B}$ ' $\left({ }^{\circ}\right)$, se hizo una comparación de los temas tratados en las dos lecciones de ' $A$ '(puntos 10.1 y 10.2) que fueron piloteadas y la lección que trata temas similares en ' $B$ ' (punto 10.3). Como las dos lecciones de 'A' tipifican las lecciones del libro, al presentar los resultados nos referimos no sólo a las lecciones de 'A' sino a ' $A$ '. Y como ' $B$ ' era la guía de la clase y del programa y por lo tanto los alumnos estaban familiarizados con él, no nos referimos sólo a la lección particular, sino a 'B'.

Se aplicó un segundo cuestionario para explorar las necesidades de aprendizaje de los estudiantes objeto del estudio y sus opiniones acerca de los dos libros. Como las dos lecciones de ' $A$ ' que fueron piloteadas enfatizan la escucha (una de las cuatro destrezas principales para adquirir una lengua; las otras tres son habla, lectura y escritura), en este cuestionario se incluyeron 2 preguntas específicas relacionadas con esta destreza. Este cuestionario, que

\footnotetext{
${ }^{1}$ Puesto que el interés de este Estudio es evaluar un tipo de libro, y no un libro en sí mismo, preferimos referirnos a ellos simplemente como ' $\mathrm{A}^{\prime} \mathrm{y}$ 'B' $^{\prime}$.
} 
consta de cinco preguntas en total, también abiertas, incluye otra sección exploratoria de los procedimientos más apropiados para trabajar con tareas y actividades en clase preferidas por los encuestados. Los resultados de estos cuestionarios se presentan en este documento dentro de las secciones correspondientes.

\section{Objetivos del programa}

Aparte de detalles específicos que el plan de estudios de una licenciatura pueda establecer, el objetivo subyacente de un programa de inglés en una Licenciatura es capacitar al estudiante para usarlo y enseñarlo en el salón de clase del Bachillerato, a nivel de las cuatro habilidades, a saber: escuchar, leer, hablar y escribir. (Entendiendo que si es capaz de usarlo en el salón de clase, lo podrá usar también fuera de él).

\section{El tipo de usuarios a quienes va dirigido el programa de inglés}

4.1 Edad de los alumnos. De los 17 alumnos encuestados, 9(52.9\%) estaban entre los 18 y los 23 años de edad, 4(23.5\%) entre los 23 y los 28, 3(17.6\%) entre los 29 ylos 33 y 1(6\%) regis tró más de 33 años.

4.2 Estrato del barrio donde vivían. Vivían en barrios de Estrato $3\{8(47 \%)\}, 4$ $\{6(35.2 \%)\}$, y $2\{3(17.6 \%)\}$, ubicados en el Norte $\{5(29.4 \%)\}$, Noroccidente $\{6(35.3 \%)$, y Sur $\{6(35.3 \%)\}$ de la ciudad de Bogotá. Esta información pudo haber servido de base para calcular el precio que un estudiante estaba en capacidad de pagar por un libro de texto de inglés. Sin embargo, independientemente del estrato al que dijeron pertenecer los estudiantes, lo que se veía era que cada vez menos de ellos compraban el libro y que para satisfacer esta exigencia del profes or, muchos lo fotocopiaban.

4.3 Porcentaje de estudiantes que trabajaban. Del grupo encuestado, 5(29.4\%) estudiantes dijeron que trabajaban y $12(70.6 \%)$ que no.

4.4 Propósitos para aprender la lengua inglesa. Los propósitos aducidos con mayor frecuencia para estudiar el inglés: fueron 'para trabajar en el campo educativo' $\{11(64.7 \%)\}$, para conseguir empleo $\{4(23.5 \%)\}$, y para desarrollarse como pers ona $\{4(23.5 \%)\}$.

4.5 Estudios de lengua inglesa adicionales a los de la Licenciatura en inglés que estaban cursando. 12(70.5\%) de los 17 encuestados señaló haber adelantado algún curso de inglés aún a nivel particular. La duración de los cursos tomados por alumnos en institutos especializados oscila, según las respuestas recolectadas, entre 2 y 17 meses. Sólo 5(29.4\%) de los 17 estudiantes dijo no haber hecho ningún curso de inglés aparte de los que incluyen los 3 semestres de la Licenciatura que estaban estudiando. 


\section{5. lidentificación de las necesidades de los usuarios}

El estudiante de Licenciatura en Español-Inglés necesita un inglés de nivel social (con énfasis en las cuatro habilidades) que, al finalizar el séptimo semestre (de los 8 que tenía la Licenciatura en el lugar donde se hizo este estudio), lo capacite para usar la lengua inglesa en el salón de clase.

Esto implica que tenga un dominio de la lengua que le permita conducir la clase en inglés, que pueda responder preguntas inesperadas de los estudiantes en relación con el tema que está viendo, que pueda conversar con ellos y dirigirles conversaciones, juegos, guiar una película proveyendo téminos, explicándolos, o diciendo qué dijo tal o cual, etc. (Lo anterior justifica que los textos para aprender tengan un buen balance de la enseñanza de las cuatro habilidades (escucha, habla, lectura y escritura).

5.1 Qué creían estos estudiantes que necesitaban aprender del inglés, adicional a lo que habían aprendido hasta el momento de realizar el presente estudio. 6(35.3\%) estudiantes cons ideraban que necesitaban aprender gramática, $5(29.4 \%)$ pronunciación, 4(23.5\%) conversación, 3(17.6\%) vocabulario, y 3(17.6\%) escucha. Llama la atención que sólo una persona $(6 \%)$, dijo que necesitaba aprender escritura, aunque la formación de todos en escritura era muy débil.

\section{Con qué concepción de "lengua" trabaja el programa de inglés donde se realizó la presente evaluación.}

De alguna manera la enseñanza del inglés en el medio colombiano, y por lo tanto en el programa donde se realizó la evaluación del texto que nos ocupa, ha estado influenciada por tres de las concepciones que han dominado la enseñanza de las lenguas: el tradicionalismo, el estructuralismo y la competencia comunicativa. El programa de inglés donde se realizó esta evaluación trabaja con esta última.

6.1 El Tradicionalismo dice que para aprender una lengua lo único que se necesita es dominar sus estructuras. Esta manera de concebir la lengua data de los siglos XVI y XVII, y seguramente se fundamentó en los objetivos de la enseñanza del Latín: hacer "una gimnasia mental..., ...un estudio sistemático y disciplinado, considerado como base para todas las formas de educación superior", según lo expresa Mallison (citado por Titone 1968:26, y luego por Richards y otro (1986:2). Encodificada en el Grammar Translation Method o método de traducción de la gramática- empezó a usarse cuando fue necesario enseñar lenguas diferentes del latín: "Los libros de texto contenían reglas gramaticales, listas de vocabulario y oraciones para traducir. Hablar la lengua extranjera no era la meta, y la práctica oral se limitaba a que los estudiantes leyeran en voz alta las oraciones que habían traducido. Estas oraciones eran construídas para ilustrar el sistema gramatical de la lengua y por consiguiente no tenían relación con las de la lengua que se usa para la comunicación oral (Richards y otro 1986:4 p. 4). 
Este método dominó en Europa entre 1840 y 1940, pero en muchos lugares de Colombia, con algunas modificaciones, es todavía común. También con algunas modificaciones, hablantes nativos diseñan todavía libros de texto para la enseñanza del inglés con un gran énfasis en este método, y muchos de nosotros, profesores, tendemos a usarlo porque no demanda mucha preparación de la clase, ni técnicas organizativas, ni mucho conocimento de la lengua inglesa.

6.2 El Estructuralismo reflejado en el audiolingualismo (ALM), comenzó a implementarse en Estados Unidos, en 1939. Para Charles Fries -su creador, y sus colegas-, 'la gramática', o 'es tructura', era el punto de partida. ...la lengua se enseñaba prestando atención sistemática a la pronunciación y a la repetición oral intensiva de sus patrones básicos. (Richards y otro op. cit. p. 46).

Acerca de este método, Stern (1983:462) señala: "Este método que tuvo su auge en Estados Unidos hasta 1966, tiene varias características distintivas: separa las destrezas: escucha, habla, lectura y escritura para su enseñanza; da primacía a lo audio-oral sobre lo escrito. El uso de diálogos es su medio principal para presentar la lengua. Enfatiza ciertas técnicas como la mímica, la memorización y la repetición de patrones".

Su mayor énfasis era en escuchar y hablar. El Audiolingualismo introdujo el laboratorio para la enseñanza de las lenguas. Como sus creadores consideraban que dominar una lengua implicaba el manejo de sus patrones gramaticales, utilizaban el Laboratorio para que los estudiantes los repitieran, con la esperanza de que escuchando y repitiéndolos 'exactamente' y con una pronunciación 'perfecta', su uso se volviera automático.

6.3 La Competencia Comunicativa, que destaca la importancia de enseñar integrando los significados y las funciones de la lengua a sus estructuras, se sintetiza en el método comunicativo. Su origen, según Richards y otro (op. cit. p. 64 ) se remonta a los últimos años 60 s. Es una aproximación para la enseñanza de las lenguas que tiene como propósitos hacer de la competencia comunicativa su metodología, y desarrolla procedimientos para la enseñanza de las cuatro habilidades que reconocen la interdependencia entre lengua y comunicación.

Este método surgió cuando lingüistas británicos comenzaron a enfatizar la necesidad de enseñar la lengua para la comunicación más que para dominar sus estructuras. Entre sus principales impulsores se cuentan Campbell and Wales, quienes afirman que no se debe considerar sólo cómo un niño adquiere competencia sistémica sino cómo desarrolla competencia comunicativa; Hymes, quien comenzó a hablar de competencia comunicativa en contraste con el concepto de competencia lingüística introducido por Chomsky; y Halliday, quien trabajó sobre el potencial comunicativo y funcional de la lengua.

El método comunicativo reconoce que la lengua no es sólo un sistema de reglas sino un recurso para la creación de significado. Que aunque nadie puede comunicarse con éxito en una lengua a menos que tenga esta competencia 
sistémica, cuando usamos la lengua la importancia de transmitir un mensaje sobrepasa la de hablar con exactitud gramatical, porque la función principal de la lengua es la interacción y comunicación.

Algunos de los principios del método comunicativo son: el sistema de la lengua sigue siendo la espina dorsal de la enseñanza, pero éste es aprendido mejor a través de un proceso de lucha para comunicarse. La contextualización de la lengua que se enseña es una premisa básica. Fluidez y lengua aceptable son la meta principal. Los estudiantes deben interactuar a través de trabajo en parejas y en grupo, o mediante sus escritos. Enfatiza las cuatro habilidades, pero da prelación a la escucha y a la lectura. Dice que la repetición puede darse pero no para conseguir el uso automático de la lengua, como un el ALM, sino, por ejemplo, para ayudar a establecer una buena entonación y pronunciación de oraciones y/o de preguntas. El Laboratorio de Idiomas también es aceptado, por ejemplo, para aprender a reconocer las palabras y el discurso de los hablantes nativos. En resumen, el método comunicativo acoge todas aquellas técnicas no sólo de los métodos aquí nombrados sino de cualquier otro, que contribuyan a enseñar/aprender la lengua para ser usada efectivamente para la comunicación, porque más que un método es una aproximación ecléctica para la enseñanza de las lenguas.

\section{Cómo se seleccionan y organizan los contenidos a ser estudiados en el programa de ingles donde se realizó la presente evaluación}

Para la selección y organización de los contenidos, el programa donde se realizó la presente evaluación trabaja con el concepto de "syllabus" y acepta la confluencia de todos aquellos syllabuses (syllabus multidimensional) que se ajusten a las necesidades de los estudiantes, dentro de una aproximación comunicativa para la enseñanza/aprendizaje de la lengua inglesa.

El Syllabus. Un Syllabus es la selección y organización de los contenidos a ser estudiados en un determinado programa. Brumfit (1984), citado por White (1988:3), a este respecto, dice: "Un syllabus es la especificación del trabajo de un departamento deteminado... organizado en subsecciones, que define el trabajo de un grupo o clase en particular".

La selección y organización se hace según la concepción que se tenga de la lengua, ya sea como un conjunto de reglas que hay que enseñar porque están ahí, o como un instrumento para la comunicación. Así, los syllabuses van desde los que centran su atención en aprender las estructuras y reglas que rigen una determinada lengua (syllabus estructurales), hasta los que enfatizan el uso de la lengua (syllabus funcionales), y partiendo de éstos aparece el multidimensional (que es con el que trabaja el programa donde se hizo la presente evaluación), que tiene en cuenta que para que un hablante dom ine el sistema de la lengua (syllabus gramatical) que quiere aprender es esencial, pero que para adquirir competencia comunicativa necesita saber en qué situaciones la usa (syllabus situacional), 
dentro de una gran variedad de tópicos (syllabus basado en tópicos) y vocabulario (syllabus lexical), y que estos propósitos se logran mejor si se trabaja con actividades que hagan reflexionar al estudiante sobre temas que motiven sus intereses, y sobre los cuales deba dar una opinión, o resolver un problema (syllabus basado en tareas).

\section{Organización de los contenidos en 'a' $y$ en 'b'}

A quiénes va dirigido el libro 'A' y de qué consta. Según su autor, 'A' es un curso de Inglés general para adultos jóvenes que los lleva desde el nivel de principiante hasta el avanzado. Utiliza el inglés británico como modelo para la gramática, el vocabulario, la ortografía, y la pronunciación, pero incluye otras variedades del Inglés para escucha y lectura.

Según información obtenida en la agencia de la editorial que lo distribuye, la serie com pleta consta de 5 libros para el estudiante. Cada libro corres ponde a un nivel. Cada nivel consta del libro del estudiante, el libro de práctica, la guía para el profesor, dos cintas de audio para el libro del estudiante, una cinta de audio para el libro de práctica, un paquete de recursos (que tuve oportunidad de ver, y contiene actividades comunicativas para la práctica de las estructuras que se van estudiando), y un libro de ejercicios. La serie incluye además un paquete de negocios.

El presente estudio se realizó con base en el libro 3, llamado "Pre-Intermediate" en la serie, que consta de 40 lecciones, el libro de práctica, y las cintas de audio correspondientes al libro del estudiante. A continuación se incluyen las características más sobresalientes de la manera como están organizados los contenidos en 'A', y cómo son tratados el vocabulario, la escucha, la conversación, la gramática y sus funciones, la lectura, la escritura, y los ejercicios del libro de práctica en el nivel examinado.

8.1 Cómo están organizados los contenidos en 'A'. Como lo dice su autor, el curso está diseñado de acuerdo con un syllabus multidimensional (Punto 7.7), que parte de la gramática y sus funciones, vocabulario, y sonidos en foma explícita, y tópicos, entrenamiento del aprendiz en competencia sociocultural, implícitam ente. Este diseño del curso en general, es el mismo de cada lección en particular.

La presentación de actividades en cada una de las destrezas (escuchar, hablar, leer y escribir) se orienta a crear en el estudiante de la necesidad de ejecutarlas para resolver un 'problema', más que para ejecutarlas porque son parte de la lección. Estos 'problemas' en muchas ocasiones incluyen textos con errores que el estudiante debe corregir mientras los lee o mientras los escucha.

A continuación se incluyen las caracterís ticas más sobresalientes de la manera como están organizados los contenidos en ' $A$ ', y cómo son tratados el vocabulario, la escucha, la conversación, la gramática y sus funciones, la lectura, la escritura, y los ejercicios del libro de práctica en el nivel examinado. 
8.2 El vocabulario. 'A' le da una gran importancia al vocabulario, aduciendo que los 'errores de gramática se toleran más fácilmente que los de vocabulario a la hora de hacerse comprender'. Esto no quiere decir que al estudiante se le pida aprender listas de vocabulario sino que éste se le presenta en el contexto de un 'problema': por ejemplo: de un conjunto de palabras seleccionar las adecuadas para corregir un texto que leyó o que escuchó; describir las cualidades que le gusta encontrar en un amigo, etc. Escuchar unas noticias para comparar si las palabras que colocó en la sección de preescucha, bajo unas categorías (ej., match, stadium, team, win, bajo la categoría 'sport', realmente corresponden a esa categoría, etc.).

8.3 La escucha. Los ejercicios de escucha en ' $A$ ' son basados en tareas: como la que se menciona arriba con respecto al vocabulario, o por ejemplo, leer un diálogo donde los participantes no están es pecificados y decidir a cuál de ellos corresponde el parlamento de acuerdo con lo que se escucha; ordenar oraciones de acuerdo con lo que se escucha, escuchar una descripción e identificar cierto tipo de palabras (por ejemplo los adjetivos usados en la descripción), etc. Al final de las 40 lecciones incluye las transcripciones correspondientes.

8.4 La conversación. Los ejercicios de conversación demandan del estudiante sus comentarios individuales o en parejas incluyendo la lengua estudiada en esa lección.

8.5 La lectura. La mayor parte de las lecciones utilizan como punto de partida una lectura que ejemplifica la lengua que quiere ser enseñada. Estas lecturas generalmente son auténticas. Pueden describir un lugar; resumir una una película; relatar un viaje; describir las costumbres alimentarias de un país; etc. $O$ pueden hablar acerca de la vida de un personaje famoso, recibir consejos para resolver una situación, para visitar una ciudad, etc. Generalmente van acompañadas de fotografías reales.

8.6 La gramática y sus funciones. El punto gramatical y/o su función aparece $(n)$ en cada lección inmediatamente después del título de ésta. Dentro de la lección hay un recuadro que las explica. Adicionalmente, al final de las 40 leciones el libro induye una sección complementaria de gramática. Hay ejercicios para práctica de la gramática vista en cada lección, prácticamente en todas las lecciones.

8.7 La escritura. En casi todas las lecciones hay ejercicios para que los estudiantes se entrenen en escritura de párrafos, con diferentes propósitos comunicativos: por ejemplo, escribir la parte final de una historia, reconstruír un texto, etc.

8.8 Los ejercicios del libro de práctica. Estos ejercicios manejan el mismo tipo de tareas que maneja el libro del estudiante, para las cuatro habilidades.

\section{Organización de los contenidos en 'b'}

A quiénes va dirigido el libro 'B' y de qué consta. Según su autor, 'B' "es un curso de Inglés general diseñado para estudiantes de Bachillerato y adultos 
jóvenes que los lleva des de el nivel de Principiante, o Falso Principiante, hasta el Intermedio".

Según información conseguida en la agencia de la editorial que los distrubuye, la Serie consta de 3 libros para el estudiante, cintas de audio para el libro del estudiante, y libro de práctica para cada uno de los libros del estudiante.

La presente evaluación se realizó con base en el estudio del libro del estudiante No. 3, o "intermedio", que consta de 40 lecciones, y sus correspondientes cintas de audio y libro de práctica. El inglés británico se usa como modelo para la gramática, el vocabulario, la ortografía y la pronunciación.

A continuación se incluyen las caracterís ticas más sobresalientes de la manera como están organizados los contenidos en 'B', y cómo son tratados el vocabulario, la escucha, la conversación, la gramática y sus funciones, la lectura, la escritura, y los ejercicios del libro de práctica en el nivel examinado.

9.1 El vocabulario. 'B' frecuentemente utiliza un vocabulario más complicado que el que utiliza Aen el libro intermedio pero al estudiante generalmente no se le plantea una necesidad crucial para usarlo.

9.2 La escucha. Las cintas de audio generalmente se limitan a recitar el texto correspondiente a cada lección. Al final incluye las transcripciones correspondientes.

9.3 La conversación. En algunas lecciones se plantean ejercicios individuales, o en parejas, para que el estudiante practique la lengua que está aprendiendo, y en otras pocas para que discuta el tema de la lección. Pero de acuerdo con la experiencia, no son temas que realmente despierten el interés del estudiante. También presenta algunos ejercicios de conversación que plantean problemas para que el estudiante resuelva, pero muchos de ellos los coloca como actividades suplementarias, en las últimas páginas del texto, y no como parte esencial de cada lección.

9.4 La lectura. Cada lección comienza con una historia o un diálogo, generalmente 'fabricados' para mostrar el tema de lengua de la lección. Pero además, de las 40 lecciones, 5 son extensas lecturas, algunas referidas a hechos ciertos, que generalmente le dejan al estudiante una enseñanza útil: por ejemplo la de un equipo de basquetball de un colegio para cuyo entrenador parece primar la amonía de un grupo que pierde en la cancha pero se divierte jugando, al éxito que pueda lograr ese mismo grupo con un sólo individuo que lo hace ganar pero que lo disocia; o las penurias de la niñez de Charles Chaplin que sin embargo no le impidieron convertirse en el más famoso actor del cine mudo en el mundo.

9.5 La gramática y sus funciones. 'B' no enfatiza las funciones por lección sino que las menciona al comienzo. Lo que más se destaca en la mayoría de las lecciones de $\mathrm{B}$ es su interés por mostrar al estudiante la estructura que quiere enseñar. 
9.6 La escritura. Además de los ejercicios de escritura que plantea en algunas lecciones del libro 3, en donde espera que el estudiante reafime la gramática y practique la descripción, dedica 4 lecciones totalmente a la escritura, yendo desde la descripción de un lugar, la narración de una his toria, la redacción de una noticia, has ta la escritura de un ensayo, para que los estudiantes los imiten.

9.7 El libro de práctica El libro de práctica frecuentemente presenta ejercicios para mecanizar la estructura o estructuras estudiadas. Estos ejercicios a veces están basados en oraciones aisladas.

10. Las lecciones piloteadas de 'a' y su comparación con las de 'b'.

Para los propósitos de la evaluación se pilotearon 2 lecciones del libro 'A' (Nos. 11 y 12), tercer libro de esa serie, para nivel intermedio, y una (No. 1) de 'B'.

Antes de seleccionar las de 'A' se hizo una revisión de las 40 del libro, para comprobar que las tipificaran. La No.1 de 'B' se especifica aquí sólo porque en ella se revisan el Presente Perfecto Simple y el Presente Perfecto Continuo, que son centrales en las 11 y 12 de ' $A$ ', Pero, en realidad, en la evaluación se comparan las de 'A' con todas las del libro 'B' porque los estudiantes lo estaban usando, y porque habían usado otros libros también de 'B' en semestres anteriores.

10.1 Lección 11 de 'A'. Esta lección presenta un diálogo entre una pareja que habla de los que tienen que hacer en un día específico. En el diálogo (escrito) varias palabras y expresiones están erradas. En la página siguiente hay un grupo de palabras y expresiones de las que el alumno puede escoger las adecuadas para corregirlo. Luego, él puede escuchar en la cinta de audio el diálogo correcto y comprobar si sus modificaciones fueron apropiadas.

(O puede utilizar la metodología que sugiere la instrucción en el recuadro, abajo).

En una sección diferente, el estudiante tiene la oportunidad de escuchar las cosas que la señora, en el diálogo, tiene que hacer, y chequear las que ya hizo. $O$ sea que en esta nueva sección el estudiante puede reforzar el vocabulario que la lección presenta, de una manera comunicativa.

A continuación se reproduce parte del diálogo, la sección de vocabulario, y aquélla donde puede chequear lo que ya hizo la señora en el diálogo, con sus corres pondientes instrucciones: 


\section{LISTENING AND VOCABULARY}

1 You are going to hear two people talking about what they've got to do. Read their conversation. Do you notice anything strange?

lan $\mathrm{Hi}$, Kate. How are you getting on?

Kate Hey, what are you doing here? I didn't expect you until later.

lan Well, I've already finished everything I had to do at work, so I thought I'd come back

and get in the way. I know you've had a relaxing day.

Kate I've had a extremely busy day, and it hasn't finished yet. Did you remember we've got Paul and Hannah for dinner tonight?

lan Yes, I did. Have you got everything you need?

Kate Well, I've already done the shopping, but I haven't sold the wine yet.

lan I'll do that. Have you dropped off the children yet?

Kate No, I haven't done that yet. They're still at school.

lan OK. Well, I'll get them when I buy the wine.

Kate No, they've got their sports club, so they'll be there until about six. You're more important here. Have you taken the car to the garage yet? It should be ready by now.

2 Listen and underline anything in the conversation that is different from what you hear.

3 Replace the words you underlined with words from the box below. There is one extra word.

busy buy clean collect help lay lie mend pick up take out throw away tidy turn on wrap

4 Look at the list of things to do. Listen and put a tick ( ) by the things Kate has already done and a cross ( ) by the things she hasn't done yet.

do the shopping take out the rubbish lay the table by the wine

collect the car start cooking clean the kitchen

throw away the

pick up the children check the bathroom

wrap up the

newspapers

turn on the heating

birthdaypresent mend the TV

Luego hay ejercicios para la práctica de la lengua objeto de la lección con las demás habilidades.

10.2 Lección 12 de 'A.'. La lección 12 comienza también con vocabulario. Provee al estudiante con una larga lista de palabras, y a continuaciòn le presenta una tabla con diferentes tipos de noticias (arte y cultura, política, deporte, etc.), y le pide colocar esas palabras en el tipo de noticia correspondiente. Después le pide escuchar seis noticias diferentes, y decidir qué tipo de noticia es cada una. 
A continuación se reproduce parte de la sección de vocabulario, y la tabla donde puede colocar cada una de las palabras de acuerdo con sus conocimientos, con sus correspondientes instrucciones:

\section{VOCABULARY AND LISTENING}

1 Look at the words in the box. In which type of news story would you expect to hear

them? Choose from the list the type of news stories below.

air force army attack bomb border bullet club currency defeat demonstration election fire gale gun hijack hostage judge jury kidnap lawyer left wing lightning lose march match missile

\begin{tabular}{|l|l|l|l|l|l|l|}
\hline & 1 & 2 & 3 & 4 & 5 & 6 \\
\hline Arts and culture & & & & & & \\
\hline Business and economics & & & & & & \\
\hline Crime & & & & & & \\
\hline Disaster & & & & & & \\
\hline Famous people & & & & & & \\
\hline Foreign Affairs & & & & & & \\
\hline Government & & & & & & \\
\hline Law & & & & & & \\
\hline Leisure activities & & & & & & \\
\hline
\end{tabular}

2 You're going to hear the six o'clock news, which has six main new stories. Listen and

decide what type of nes story each one is. Put a tick ( ) in the appropriate boxes in the chart. Some stories may be of more than one type.

El resto de la lección incluye secciones similares a los de la anterior (entre otras, un ejercicio donde el estudiante debe corregir errores y luego escuchar la versión correcta para saber si su trabajo es tá bien hecho).

10.3 Lección 1 de ' $B$ '. Después nos remitimos a la lección 1 del libro ' $B$ ', -guía de la clase en ese semestre, y tercero en la Serie que el Programa estaba usando en el momento de hacer la evaluación-, en donde los temas del Presente Perfecto Simple y Presente Perfecto Continuo son revisados, y que hacía días habíamos vis to en clase.

A continuación se reproduce la parte de la Lección 1 que trata este tema, con sus correspondientes ins trucciones: 
D Read these questions and answers from an interview with Pepe Ashton. Then work with a classmate. Student A ask B the questions in the box. Student B imagine you are Pepe. Find the answers below:

Example:

A: Where do you live?

B: In Australia

Present simple

1 Where do you live?

2 Do you like travelling?

3 What do you do in the circus?

Present continuous

4 Where in Australia is the circus performing at the moment?

5 What new circus act are you learning at the moment?

6 Are you revising for your exams?

Present perfect simple/continuous

7 Have you always lived in a caravan?

8 Have you ever had a serious accident?

9 How long have you been working in the circus?

10 How long has your family been running a circus?

Answers

Since I was five years old. In northern Queensland Yes, it's great.

No, not really. I've fallen lots of times, but l've never hurt myself badly.

Yes, I am. I've got them in two weeks. The flying trapeze - My father's teaching me.

For nearly 150 years. In Australia. I'm an acrobat

Yes, we've never lived in a house because we're always on the road.

- Now listen to the interview and check your answers.

Nota: la negrilla es del autor de 'B'

En la segunda columna de esa misma página hay una foto de Pepe. Después hay un ejercicio en donde se le pide al estudiante que cierre el libro y diga lo que recuerda acerca de Pepe.

En seguida otro en donde dos estudiantes deben intercambiar preguntas basadas en una lectura, finalmente una actividad de escritura. Después de revisar esta Lección, se aplicó el siguiente cuestionario:

Cuál de los dos libros le gusta más y por qué: De 17 respuestas sólo 2(11.8\%) se refineron a a ' $B$ '. 
Las dos hablan positivamente de este libro. Pero en $12(70.6 \%)$ respuestas más dijeron que les gustaba ' $A$ '. Estas 12 razones se pueden categorizar en tres criterios: les gustaba porque les ayudaba a mejorar el inglés (4), les exigía un mayor compromiso: pensar, analizar, discutir $\{4(23.5 \%)\}$, maneja las cuatro destrezas en forma simultánea $\{2(11.8 \%)\}$.

En otras 3 respuestas (17.6\%) (dijeron que les gustaban los dos ('A' y ' $\mathrm{B}$ ') porque uno se complementaba con el otro. En otras respuestas de estos tres alumnos $(17.6 \%)$ dijeron que les gustaban los dos libros, también hubo una (6\%) en que dijeron que les gustaba 'A' porque los ponía a pensar.

Qúe opina de las lecciones de escucha de 'B': En 11(64.7\%) de las respuestas expresaron puntos positivos de las lecciones de 'B' y en $5(29.4 \%)$ expresaron puntos negativos. Dos de los puntos negativos señalaban el hecho de que estas lecciones no les daban oportunidad para pensar. Una persona no respondió.

Qué opina de las lecciones de esucha de ' $A$ ': 13 personas $(76.4 \%)$ dijeron que les gustaban las lecciones de 'A' y sólo $4(23.5 \%)$ dijeron que no. Uno $(6 \%)$ de estos comentarios se refería a que son comprensibles y dinámicas; otro (6\%) a que les permitían participar activamente; otro más (6\%), destacaba su realismo ("llevan más al debate, ya que no son ejercicios de simples conversaciones sino temas que vemos a diario"); y otro (6\%) a que eran útiles para avanzar en el idioma. Tres (17.6\%) de las respuestas se referían a que son más complicadas que las de 'B'.

Al preguntárseles directamente acerca de la escucha, en cada uno de los dos libros, la preferencia por ' $A$ ' también fue contundente (76.4\%). Además, lo que observé durante la sesión de escucha que realicé con las lecciones de 'A', creo que hubiera sido irrebatible para cualquier profesor: los estudiantes escucharon el diálogo de la lecciòn 11 (punto 10.1) para comprobar que lo que habían escrito estaba correcto. Luego procedimos a escuchar la lección 12 (punto 10.2). Al final de las instrucciones para realizar este ejercicio, el autor sugiere que varias noticias pueden corresponder a más de una categoría ('política' y 'gobierno', por ejemplo, ó 'crimen' y 'desastre').

Después que escucharon y comencé a preguntar bajo qué categoría habían colocado cada noticia, sólo bastó que un estudiante enunciara una para que muchos otros gritaran espontáneam ente que esa no era la correcta, o que estaba de acuerdo, etc. Era tanto el entusiasmo que no se quedaron sentados sino que se pusieron de pié aún los más calmados. Yo les pedía que hablaran sólo en Inglés aunque cometieran errores. Todos querían hablar y luchaban por enconrar las palabras para hacerse entender. Fue una clase emocionante. Al final, algunos se quedaron para decime que llevara más ejercicios como ése.

En contraste, como en 'B' lo más notorio es oír el texto de la lección (sin querer decir que es té totalmente desprovisto de ejercicios que hagan razonar y participar al alumno), nunca he visto una reacción parecida frente a una lección de escucha, 
o de otra destreza, por parte de estos estudiantes. Tampoco desconozco el hecho de que los estudiantes suelen reaccionar en una forma más favorable ante materiales distintos a la guía de la clase, porque generalmente les planteo materiales variados, sobre todo para la escucha.

\section{PROCEDIMIENTOS PARA TRABAJAR CON TAREAS Y ACTIVIDADES EN CLASEPREFERIDOS POR ESTOS USUARIOS}

11.1 Cuáles son las formas más útiles para aprender el inglés: El análisis de las respuestas a esta pregunta pemite concluír que estos estudiantes consideraban que la forma más útil para aprender el inglés era practicándolo $\{12(70.5 \%)\}$; utilizando medios audiovisuales $\{4(23.5 \%)\}$, escuchando $\{3(17.6 \%)\}$, es cribiendo $\{2(11.8 \%)\}$, utilizando canciones $\{2(11.8 \%)\}$. Otras respuestas $\{7(41.1 \%)\}$ se refieren a 7 diferentes temas.

11.2 Qué es lo mejor que le puede brindar un material par el aprendizaje de una nueva_lengua: $4(23.5 \%)$ de estas respuestas aluden a la gramática, y otras 4 $(23.5 \%)$, a la claridad en la presentación de los temas. $3(17.6 \%)$ más a su funcionalidad en términos, por ejemplo, de qué tan útiles son los temas y qué tan asimilables. El resto de las respuestas se da en porcentajes menos significativos.

\section{CONCLUSIONES Y RECOMENDACIONES}

A continuación se presentan las conclusiones y recomendaciones derivadas de este estudio de acuerdo con los criterios tenidos en cuenta para evaluar el texto usado aquí como ejemplo.

1. Objetivos del programa. El libro evaluado satisface los objetivos del programa en donde se realizó la evaluación, entre otras razones mostradas a través del estudio, porque presenta los contenidos en un syllabus multidimensional (Puntos 7 y 8.1) y porque facilita la ens eñanza comunicativa de la lengua (Puntos 6.3 y 8.1 a 8.6).

2. Edad de los alumnos. Todos los alumnos eran adultos, lo cual permite concluír que en las ilustraciones de un libro de texto para este tipo de alumnos deberían primar las fotografías sobre las caricaturas; y que el nivel de complejidad de la presentación de las unidades de aprendizaje en términos de ambientes sociales, temas, vocabulario, y presentación de los mismos, debe ser apropiado para adultos que están recibiendo una formación universitaria de carácter pedagógico. (Punto 4.1).

3. Estrato socioeconómico. El hecho de que los estudiantes objeto de este estudio hayan dicho pertenecer principalmente a estratos 3 y 4 indica que posiblemente estaban en capacidad de adquirir un libro para el estudio del inglés cuyo cos to os cilara entre $\$ 30$ y 40.000 Punto 4.2$)^{2}$

\footnotetext{
${ }^{2}$ Al momento de escribir este artículo no se encontró en el DANE un estudio que muestre la relación entre estrato socioeconómico e ingresos.
} 
4. Actividad laboral. El hecho de que la mayor parte de los estudiantes del grupo encuestado hubiera dicho que no trabajaba, permitía suponer que estaban en capacidad de concentrarsu atención en el aprendizaje y por lo tanto obtener mejor provecho del texto de inglés (Punto 4.3).

5. Propósito para estudiar la lengua inglesa. El propósito principal para estudiar el inglés citado por ellos era instrumental: "para conseguir empleo en el campo educativo", seguido por el de "desarrollarse como persona". Por lo tanto se requería un texto que contribuyera a capacitarlos para enseñar inglés a través del inglés, en un contexto de adquisición de valores (Punto 4.4).

6. Formación en lengua inglesa. La circunstancia de que 12 de los 17 estudiantes que formaron parte del estudio hubieran dado cuenta de haber tomado cursos de inglés adicionales a los de la Licenciatura permite concluír que eran personas interesadas en aprender la lengua inglesa pero, además, que el libro que se escogiera no debía ser tan complejo como para que los que no hubieran tomado cursos extras no pudieran acoplarse a él, ni tan fácil como para que los que tenían mejor nivel perdieran el interés. (Punto 4.5).

7. Habilidades necesarias para aprender una lengua. Entre 16 de los 17 estudiantes se menciona la mayor parte de las habilidades necesarias para aprender una lengua (gramática, pronunciación, conversación, vocabulario y escucha), pero sólo uno de los 17 menciona la escritura (Punto 5), a pesar de la formación tan débil que mostraban en esta destreza. Ninguno mencionó la lectura. El hecho de que sólo uno hubiera nombrado la escritura puede significar que en las oportunidades que ellos habían tenido para aprender la lengua inglesa, a la escritura no se le había dado el énfasis necesario y por eso ellos no habían aprendido a apreciar el valor de esta destreza. Como esta es una situación común a muchos ambientes de aprendizaje de la lengua inglesa, ya que según Byrne (1991), por una parte, es posible aprender a hablar sin aprender a escribir $y$, por otra, la escritura es la destreza que menos usa el común de las personas, el texto que se escoja para formar futuros profesores de inglés debería incluír un buen balance de la enseñanza de las 4 habilidades, puesto que ellos sí necesitan dominarlas todas. Esta recomendación cobija por supuesto la lectura, no mencionada por los estudiantes encuestados, pero base para la enseñanza de la escritura y proveedora importante de vocabulario y estructura para aprender a hablar.

8. Tipo de libro de texto que preferíían. Los estudiantes preferirían un libro del tipo de "A" para aprender la lengua inglesa porque les ayuda a mejorar el Inglés, les exige pensar, analizar, discutir, y porque maneja las cuatro habilidades en forma simultánea (Punto 10). Este planteamiento está de acuerdo con la idea de syllabus multidimensional (Punto 7) aceptada por el programa donde se realizó el presente estudio, y punto 8.1 organización de los contenidos en "A", según su autor).

La preferencia por un libro del tipo de "A" es también manifiesta en las opiniones sobre 
la escucha en particular, tanto en "A" como en "B". El $76.4 \%$ favorece a "A" y el $64.7 \%$ a "B".

9. Ningún libro es la panacea. La preferencia por un libro del tipo de "A" no excluye la necesidad de usar otros textos ya que como lo anotan algunos estudiantes (Punto 10), no es conveniente "...encasillarse en un solo libro cuando se trata de aprender".

\section{BIBLIOGRAFIA}

Dubin, Fraida \& other, 1986. Course Design. Developing Programs and materials for Learning. CUP.

Forero, Gladys, 1996. The Teaching of Listening through Task based Activities. with Special reference to Pre-service Teachers of English in Colombia. (Thesis) Bogotá, 1996.

Neville, Grant, 1987. Making the most of your textbook. London, Longman.

Nunan, David, 1989. Designing Tasks for the Communicative Classroom. CUP.

Nunan, David, 1988. Syllabus Design. OUP.

Prabhu, N. S., 1987. The Bangalore Project. Hong Kong.

Richards \& Rodgers, 1986. Approaches and Methods in Language Teaching. CUP

Sheldon, Leslie E. (eds.), 1987. ELT Textbooks and Materials: Problems in Evaluation and Development. ELT Dct., The British Council.Stern, H. H., 1983. Fundamental Concepts of language Teaching. OUP.

White, Ronald, 1988. The ELT Curriculum. Design, Innovations and Management. Basil Blackwell.

Wilkins, D.A., 1976. Notional Syllabus. OUP. 